\title{
Towards \\ Architectural Foundations for Cognitive Self-aware Systems
}

Ricardo Sanz

Universidad Politécnica de Madrid Autonomous Systems Laboratory
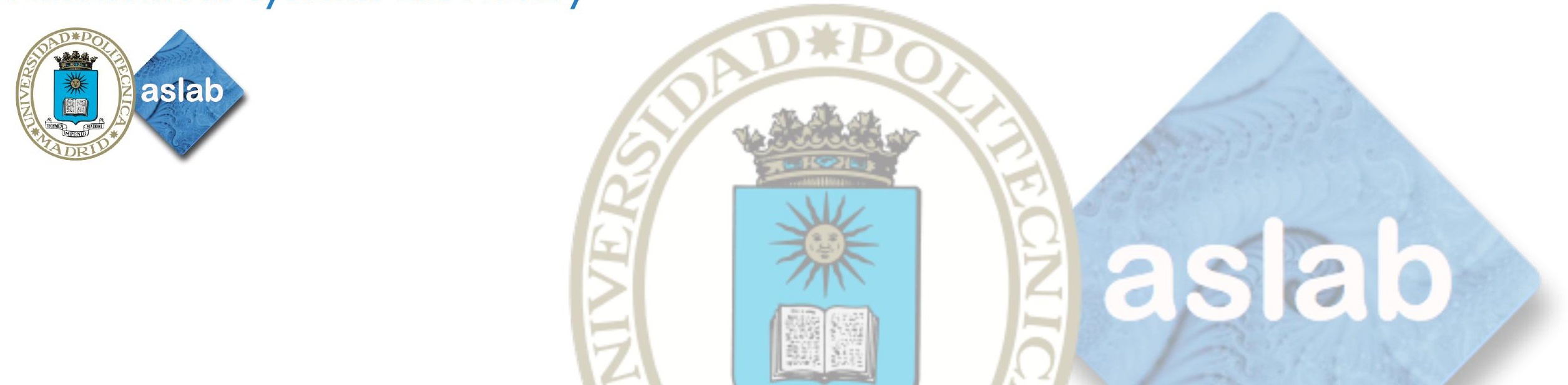


\section{Abstract}

-The BICA 2012 conference main purpose is to take a significant step forward towards the BICA Challenge -creating a real-life computational equivalent of the human mind. This challenge apparently calls for a global, multidisciplinary joint effort to develop biologically-inspired dependable agents that perform well enough as to to be fully accepted as autonomous agents by the human society. We say "apparently" because we think that "biologically-inspired" needs to be re-thought due to the mismatch between natural and artificial agent organization and their construction methods: the natural and artificial construction processes. Due to this constructive mismatch and the complexity of the operational requirements of world-deployable machines, the question of dependability becomes a guiding light in the search of the proper architectures of cognitive agents. Models of perception, cognition and action that render self-aware machines will become a critical asset that marks a concrete roadmap to the BICA challenge.

-In this talk we will address a proposal concerning a methodology for extracting universal, domain neutral, architectural design patterns from the analysis of biological cognition. This will render a set of design principles and design patterns oriented towards the construction of better machines. Bioinspiration cannot be a one step process if we we are going to to build robust, dependable autonomous agents; we must build solid theories first, departing from natural systems, and supporting our designs of artificial ones. 


\section{Content}

I. Rethinking "The Challenge"

2. Cognitive Systems Biological Insights

3. Self-aware Systems

4. Architectural Foundations of SAwS

5. Example Implementation 


\section{Rethinking \\ "The Challenge"}




\section{The BICA Challenge}

"Creating a real-life computational equivalent of the human mind."

This challenge apparently calls for a global, multidisciplinary joint effort to develop biologicallyinspired dependable agents.

- Agents that perform well enough as to to be fully accepted as autonomous agents by the human society.

... apparently 


\section{The brain modules trap}

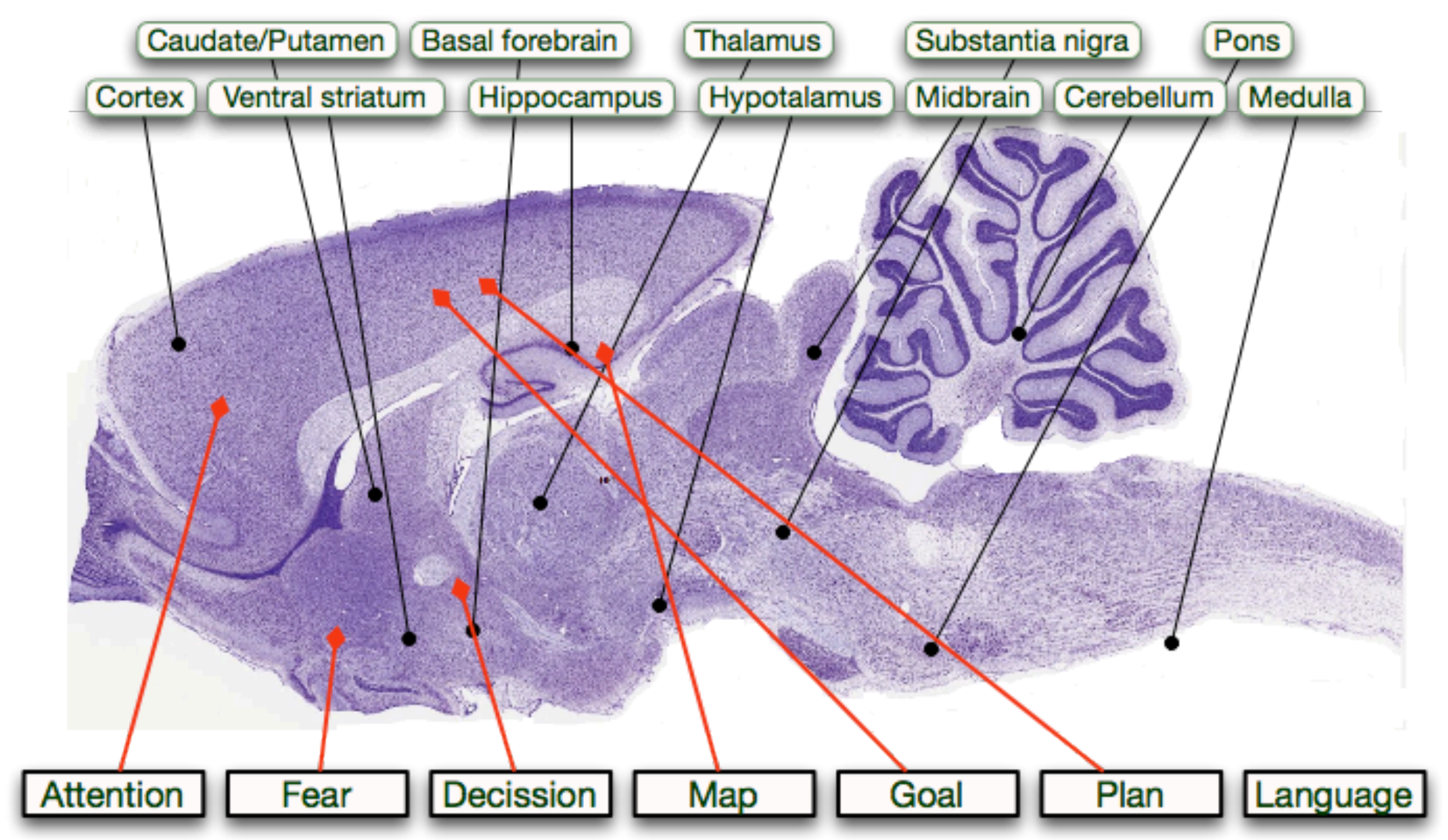




\section{Rethinking "biologically inspired"}

"Biologically-inspired" needs to be re-thought due to the mismatch between natural and artificial agent organization and their construction methods.

Due to the complexity of the operational requirements of world-deployable machines, the question of dependability becomes a guiding light in the search of the proper architectures of cognitive agents.

Models of perception, cognition and action that render self-aware machines will become a critical asset that marks a concrete roadmap to the BICA challenge. 


\section{Beware the constructive mismatch}

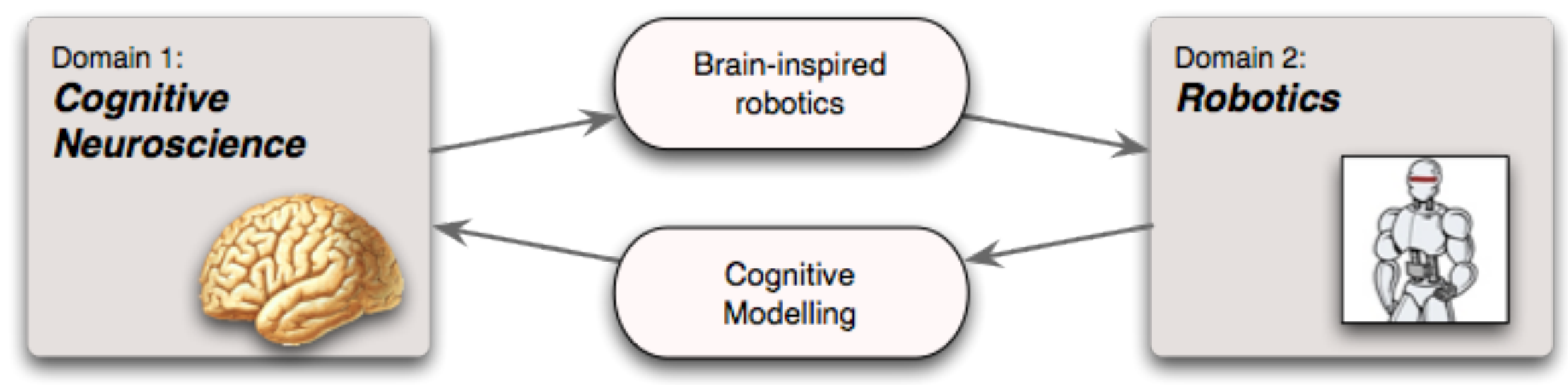




\section{The ASys Strategy}

We need a methodology for extracting universal, domain neutral, architectural organizations from the analysis of biological cognition.

This will render a set of design principles and design patterns oriented towards the construction of better, more robust machines.

- Bio-inspiration cannot be a one step process; we must build solid theories first, departing from natural systems, being general and supporting our designs of artificial agents. 


\section{Build theories first}

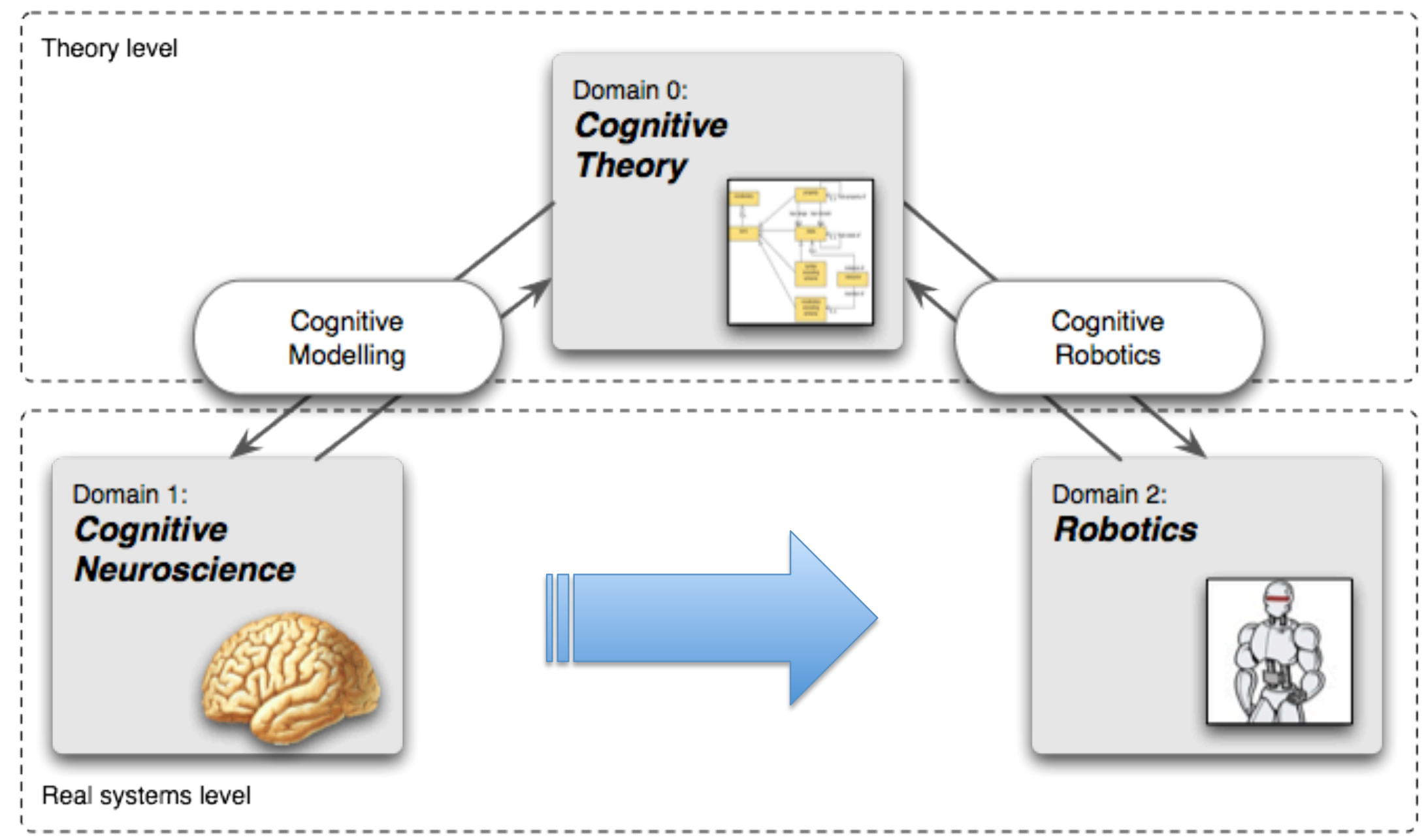




\section{Cognitive Systems Biological Insights}




\section{The agent models the world}
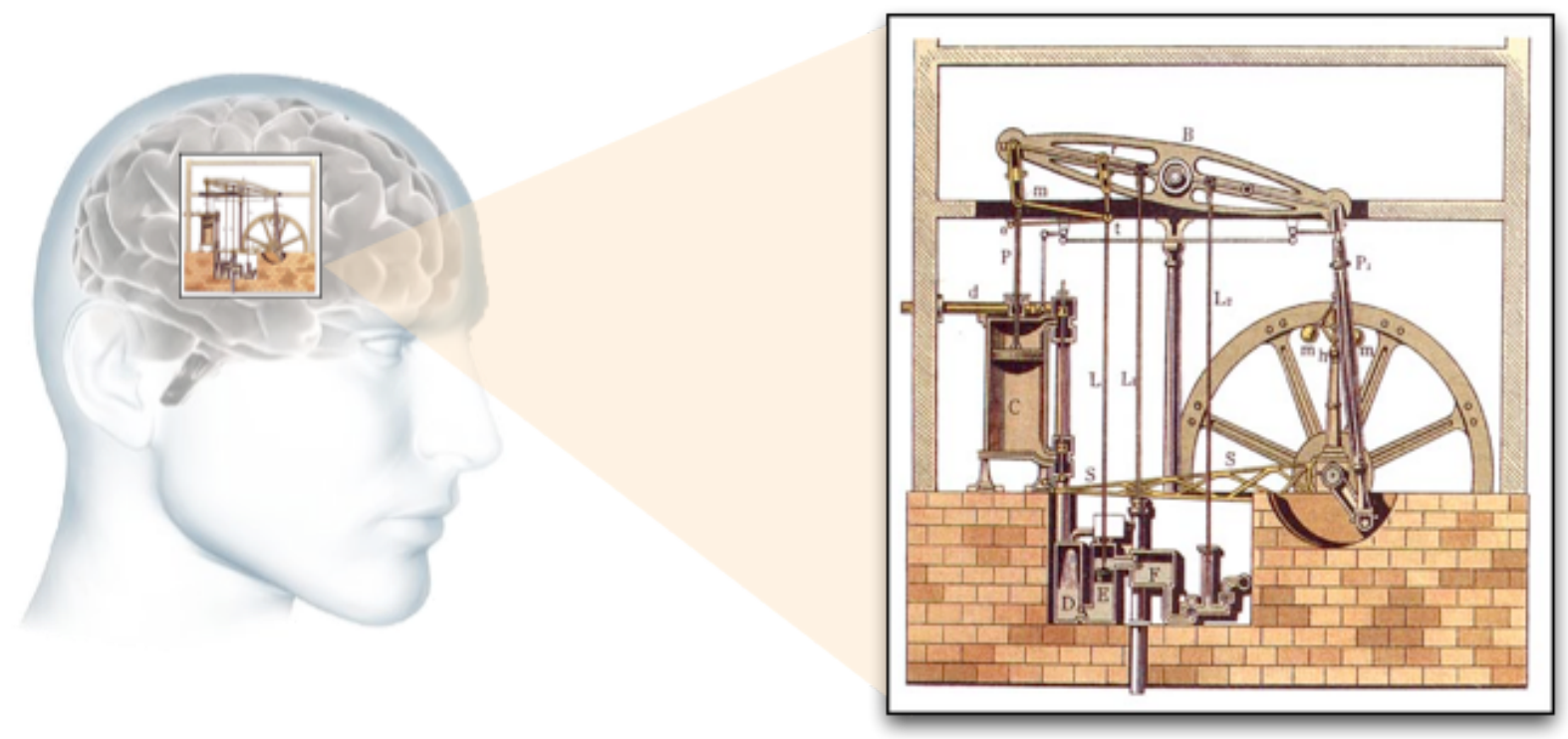

Models are not just photographs.

They are functionally equivalent dynamical systems. 


\section{Cognitive Systems Insights}

- Cognition is a control mechanism that enables body efficacy in a complex environment

- This efficacy is achieved internalizing a model of the environment, the agent and their relation

$M($ agent $)+M($ environment $)+M(R($ Agent,Environment $))$

- The agent integrates and uses the models to compute actions, anticipating their effects and hence their value

Minds are model-predictive, integrated controllers 


\section{Models and reality}

Using George Box words:

"all models are false; some are useful".

They are used by means of execution engines to:

- Predict

Retrodict

Invert (computing control actions)

etc.

Models and engines may be collapsed into single components 


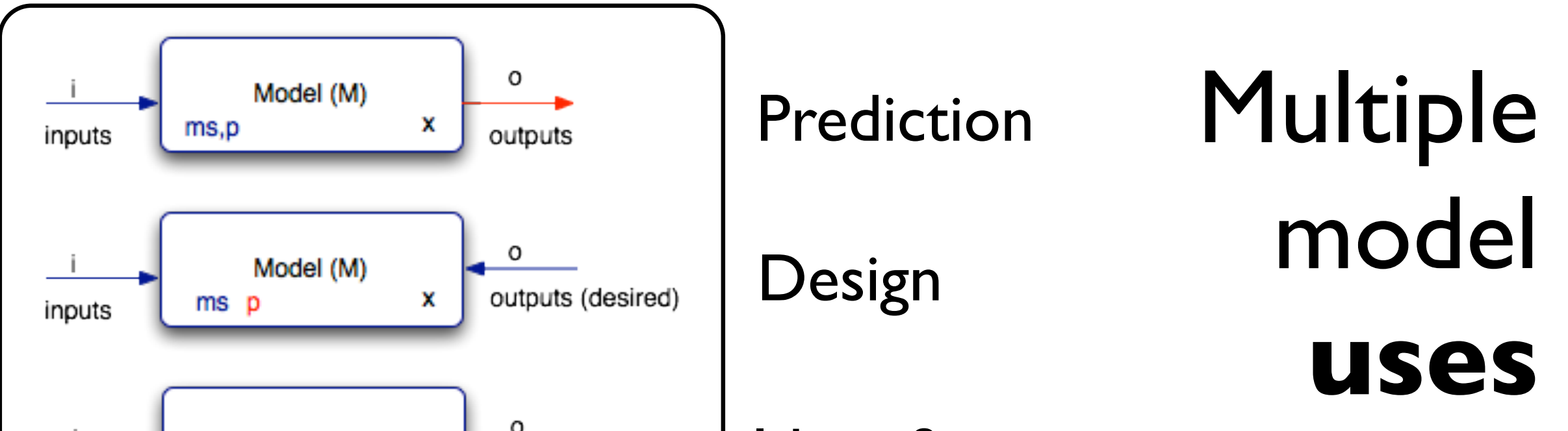

Identification

Control

Diagnosis

Optimisation 


\section{Universal Cognition Design Principles}

- The application of the generalization doctrine to the analysis of the problem of construction of a general cognitive controller has lead us to the formulation of several design principles.

- These principles can be used in two ways:

- For the construction of reusable assets for a product line approach to robust autonomous systems.

As theoretical analyses of natural cognition 


\section{Principles}

Model-based cognition: A cognitive system exploits models of other systems, of self, in their interaction with them.

Model isomorphism: An embodied, situated, cognitive system is as good performer as it is able to keep its models isomorphic to realities.

Anticipatory behavior: Agents maximise timely performance by means of anticipatory (predictive) models. 


\section{Principles}

- Unified cognitive action generation: Agents generate action based on an integrated, scalable, unified model of task, environment and self in search for global performance maximisation.

- Model-driven perception: Perception is realized as the continuous update of the integrated models used by the agent by means of real-time sensorial information. 


\section{Principles}

System awareness: An aware system is continuously perceiving and generating meaning -future value- from the continuously updated models.

System self-awareness: A self-aware system is continuously generating meanings from continuously updated self-models in a model-based cognitive control architecture. 


\section{Self-aware Systems}




\section{Self-Aware Autonomous Control Systems}

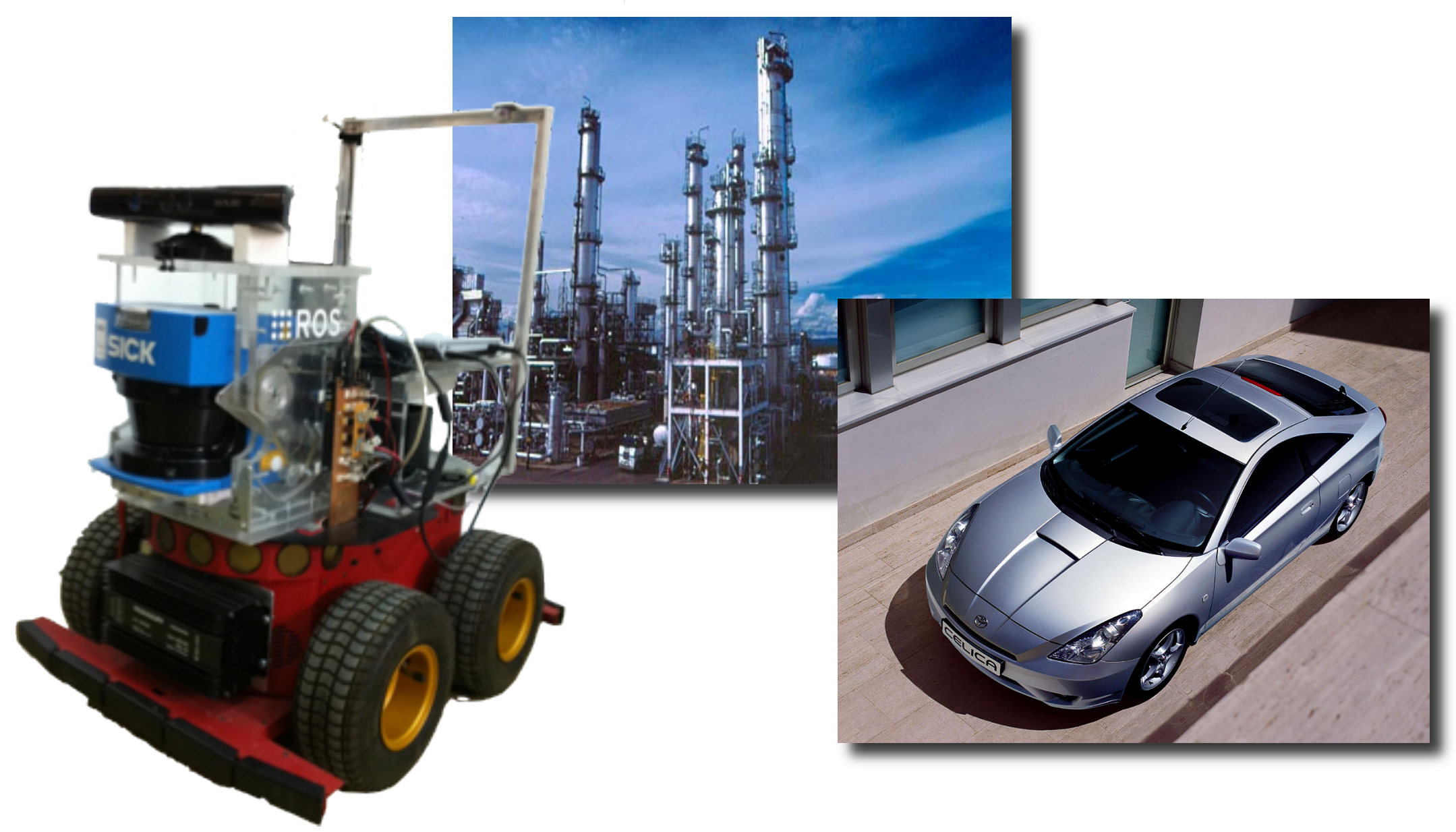




\section{Self-control and model}

[Conant and Ashby 1969]

Every good controller shall internalise a model of the controlled plant.

- Cognitive science reading:

Self-representation is necessary for proper behavior.

A system with a self-model can better control its own behavior 


\title{
Agent access to Self-Body
}

Leverage model-based engineering of the system

\author{
Use the \\ engineering models \\ as run-time \\ self-models
}

The agent becomes self-aware

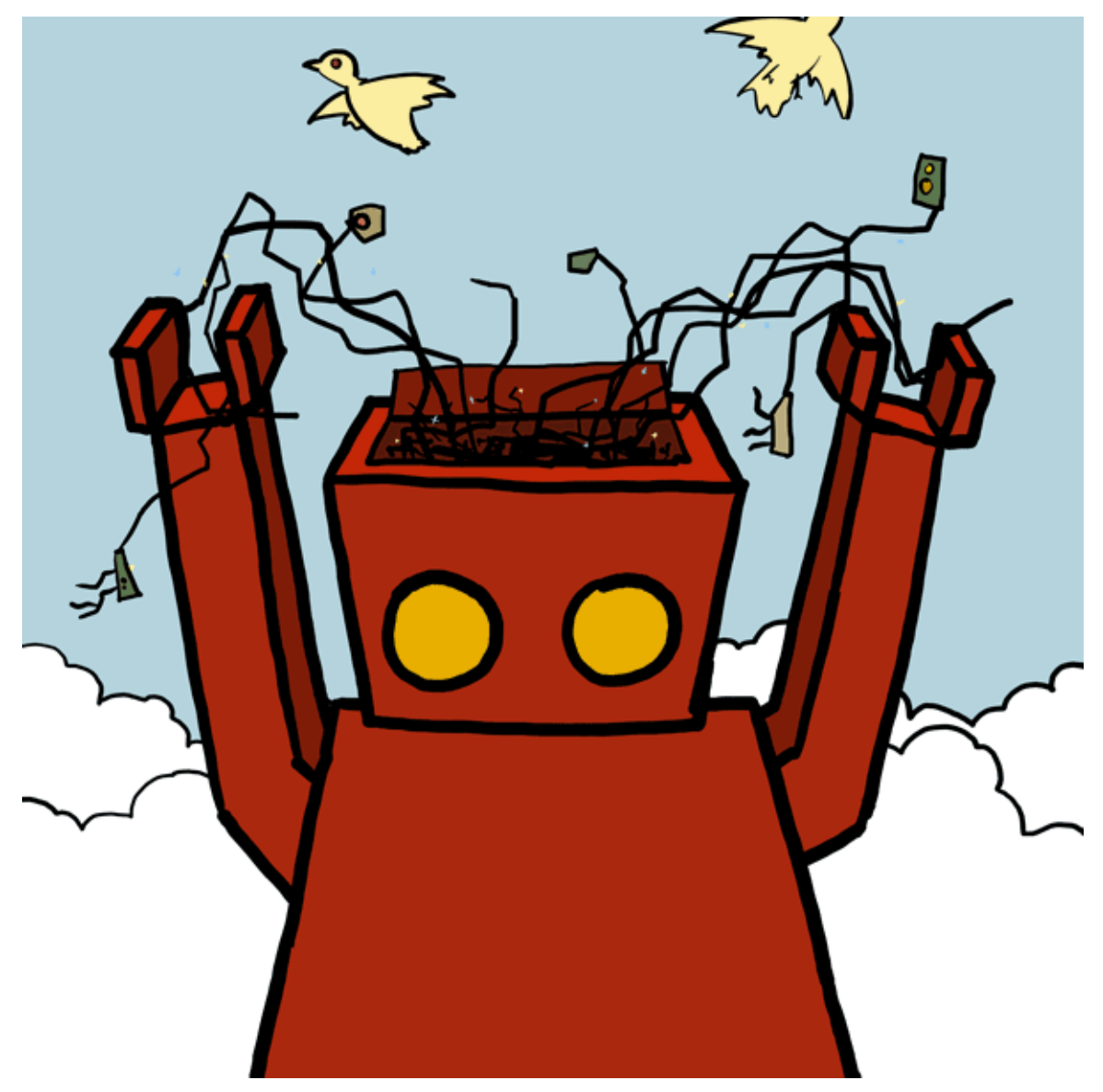




\section{Machine Consciousness?}

- The approach based on model-based systems engineering can be described as explicit model-based, reflective, predictive, adaptive autonomous systems engineering.

The key is using the engineering models as selfmodels

- The major value is that autonomous control based on selfmodels enables an increased awareness that can lead to more robust performance

Provides a road to both:

I) expressing an unified theory of consciousness and

2) using it to build machines 


\section{Architectural Foundations}




\section{Architecture and Self}

The system self-models shall essentially capture the system architecture

- Architecture encompasses the set of significant design decisions about the organization of a system

Structure as elements and their interfaces by which a system is composed

- Behavior as specified in collaborations among those elements

- Composition of these structural and behavioral elements into larger subsystems

Architectural style that guides this organization

[Booch] 


\section{Architecture defined}

There is a "Formal Definition" of architecture

IEEE |47|-2000

- Software architecture is the fundamental organization of a system, embodied in its components, their relationships to each other and the environment, and the principles governing its design and evolution.

- ISO/IEC 420 10:2007, Systems and Software Engineering Recommended practice for architectural description of softwareintensive systems. 


\section{Choose the right description level}

What is the right description level ?

statement/rule/neuron

procedure/kb/nn

module ...

pattern ...

architecture ...

- We choose the design pattern level as the proper level for system description, analysis and construction

- The rationale for this election is their function-centric nature and intrinsic composability 


\section{What is a design pattern?}

A mini-architecture, incomplete but useful

A partial organization of systems components that focus on a specific aspect

- Typically associated to a concrete system function or system property

Also related to processes

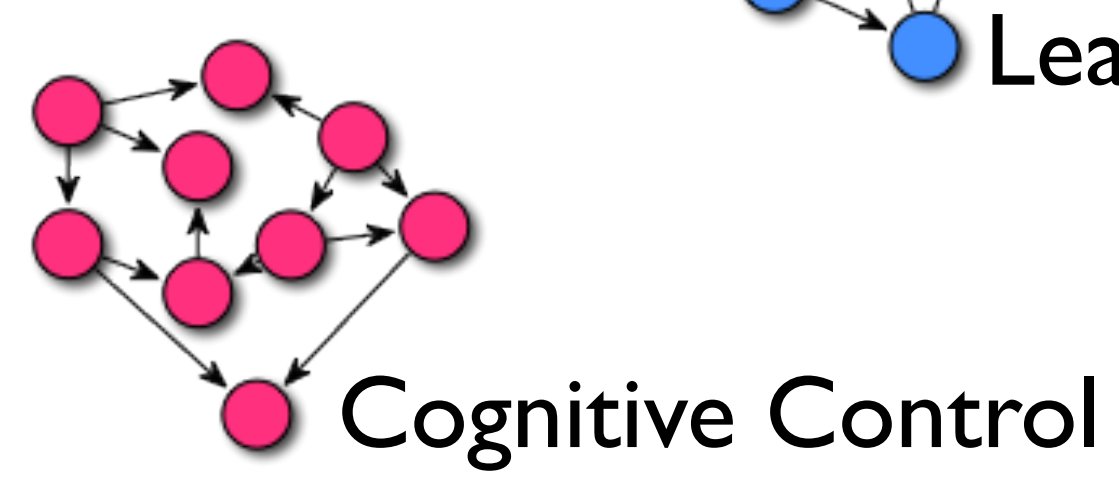




\section{The PEIS Pattern}

\section{Physically Embedded Intelligent System}

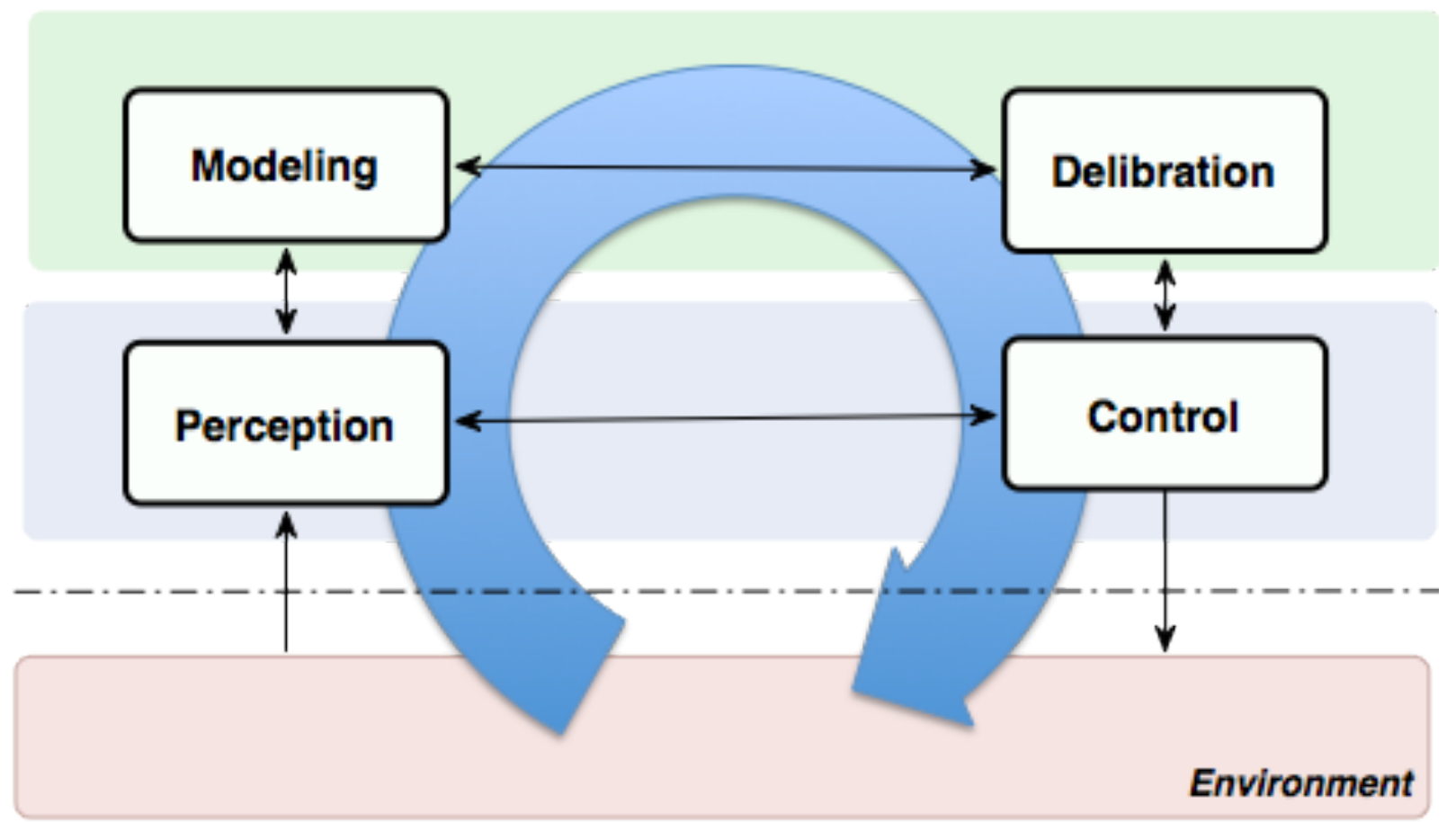

[Saffiotti] 


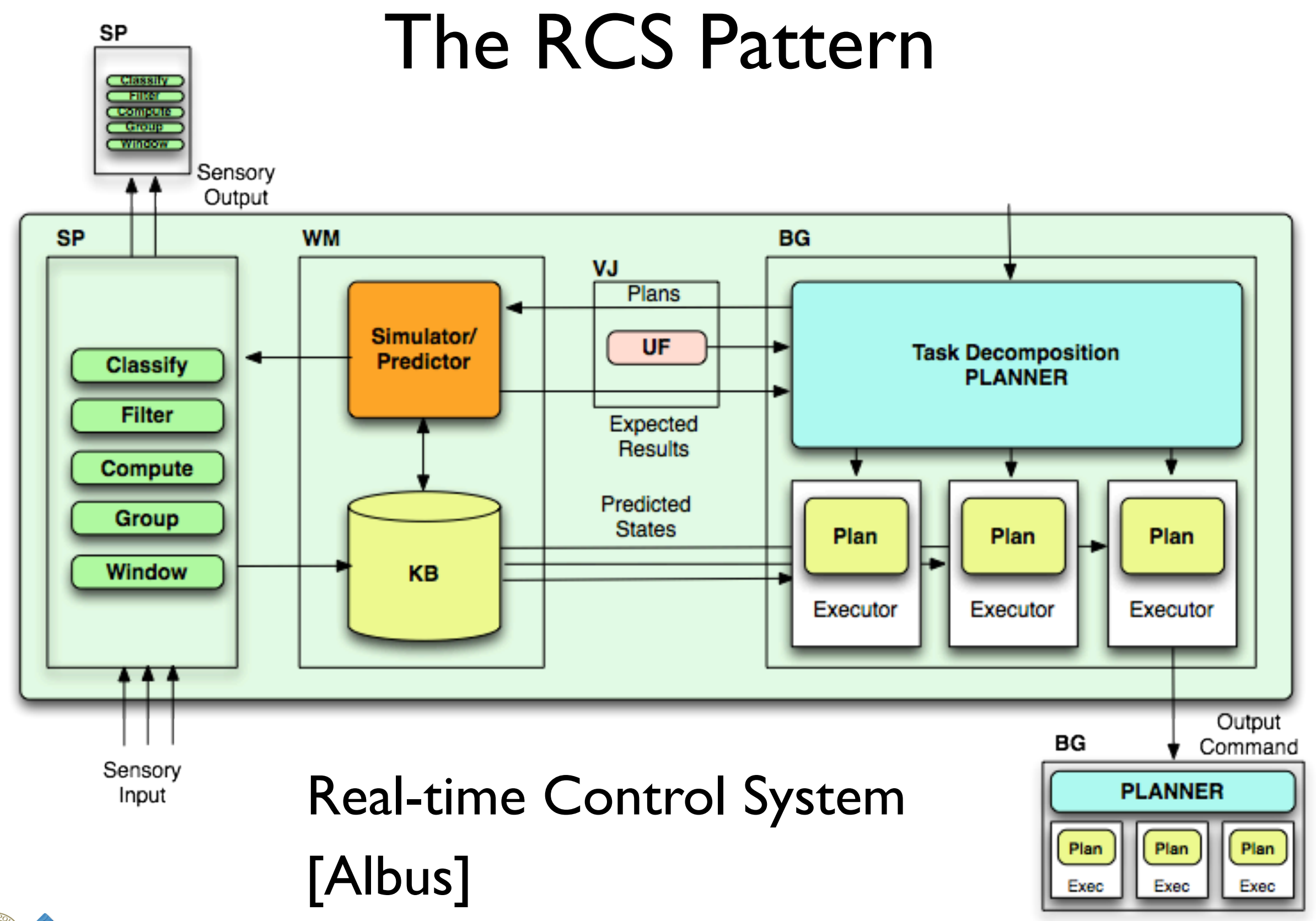




\section{The ASys Patterns}

We try to identify and re-think biological patterns -esp. at the cognitive level- to define general patterns ofr autonomous systems engineering

Process patterns:

Captured in the ASys engineering process

System patterns

To guide the design and organisation of the autonomous system

Examples: the ECL, the MC, the DMR ... 
The ASys Process Patterns

- Patterns can describe processes

The ASys Process:

- Autonomous self-aware systems can be built by model transformation

The agent Synthesis pattern is pattern-driven and exploits an asset base

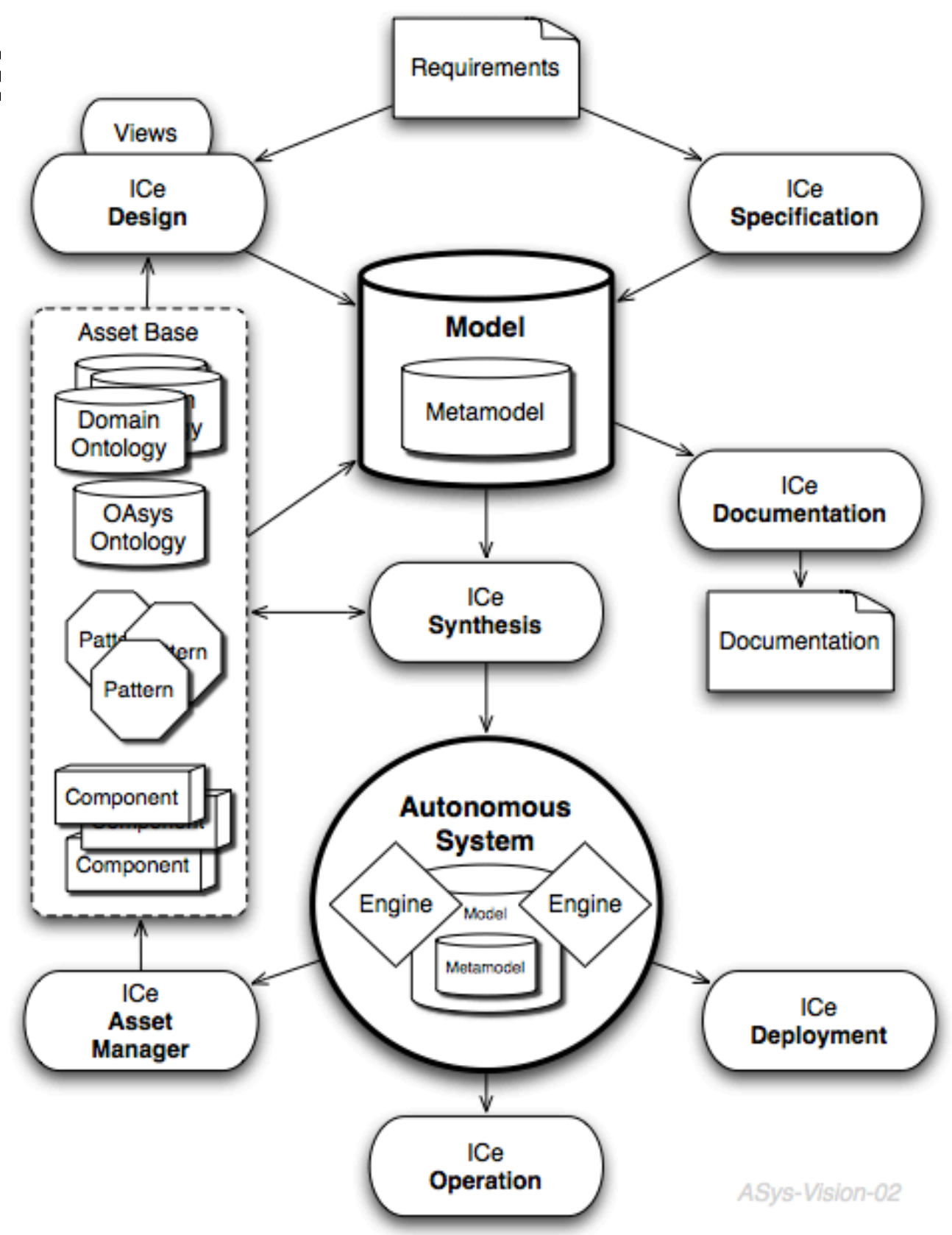




\section{The ECL Pattern}

Knowledge $=$ Model

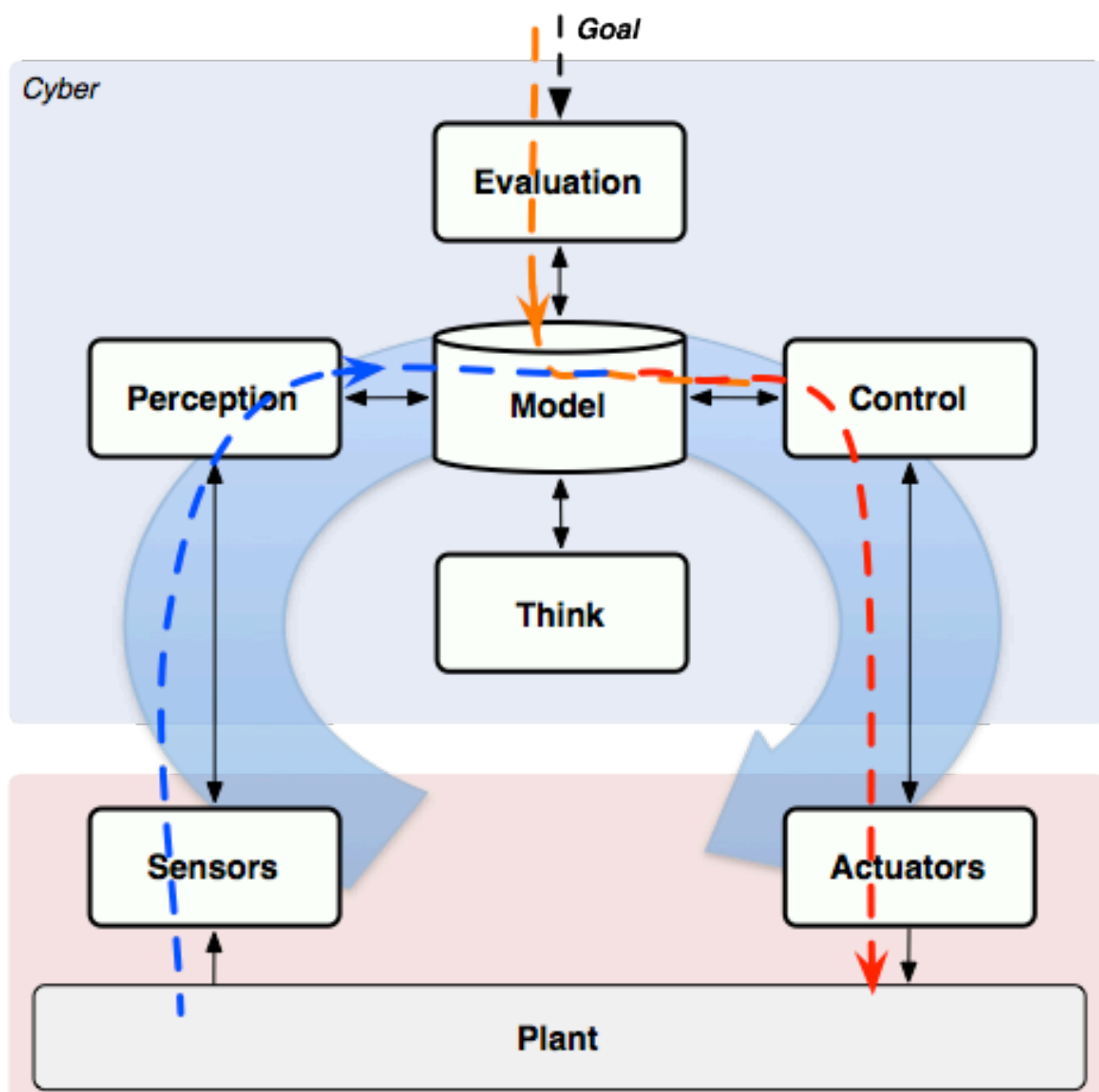




\section{The MC Pattern}

- The MetaControl pattern captures the capability of self-observation and control

- The agent sees itself as a behaving -active- entity

- Notice that metacontrol is different from nested control (Layers pattern)

They differ in the

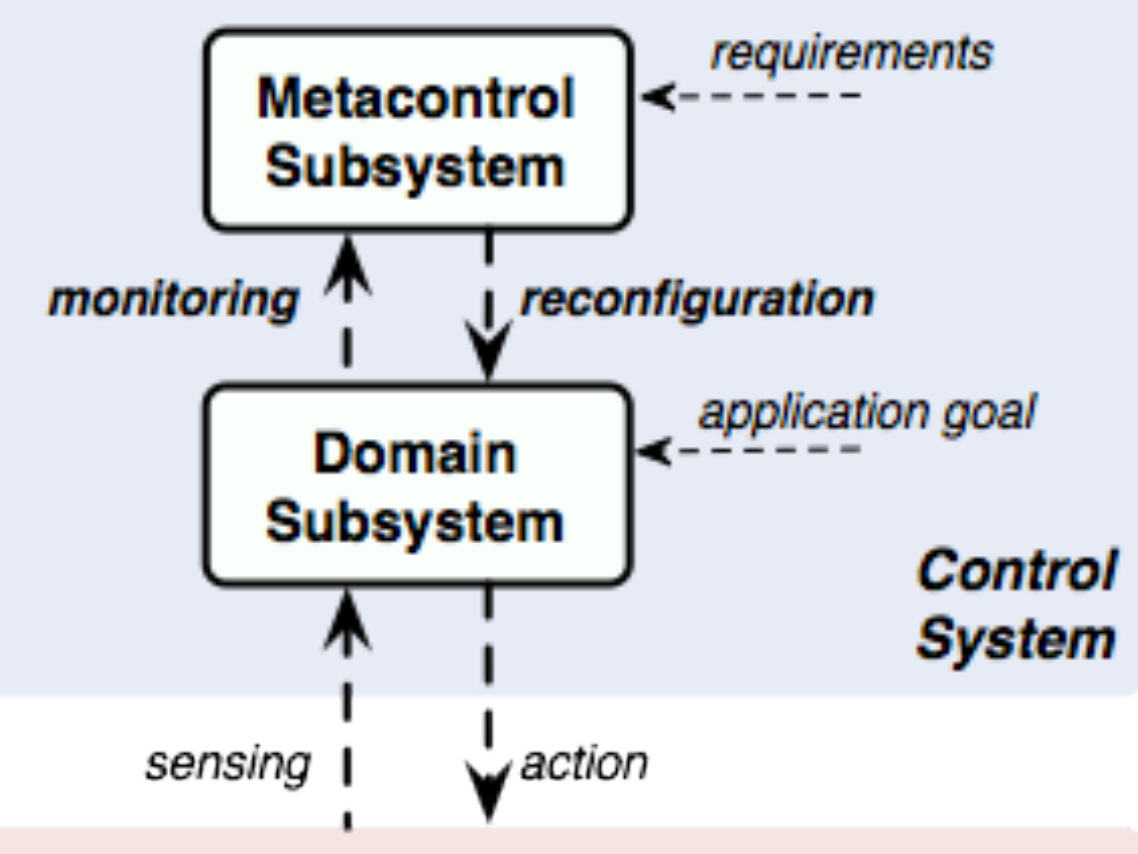
domains of control 


\section{The DMR Pattern}

- The Deep Model Reflection (DMR) pattern exploits the deep models used in model-driven systems engineering

- It enables agent deep architectural reflection during runtime

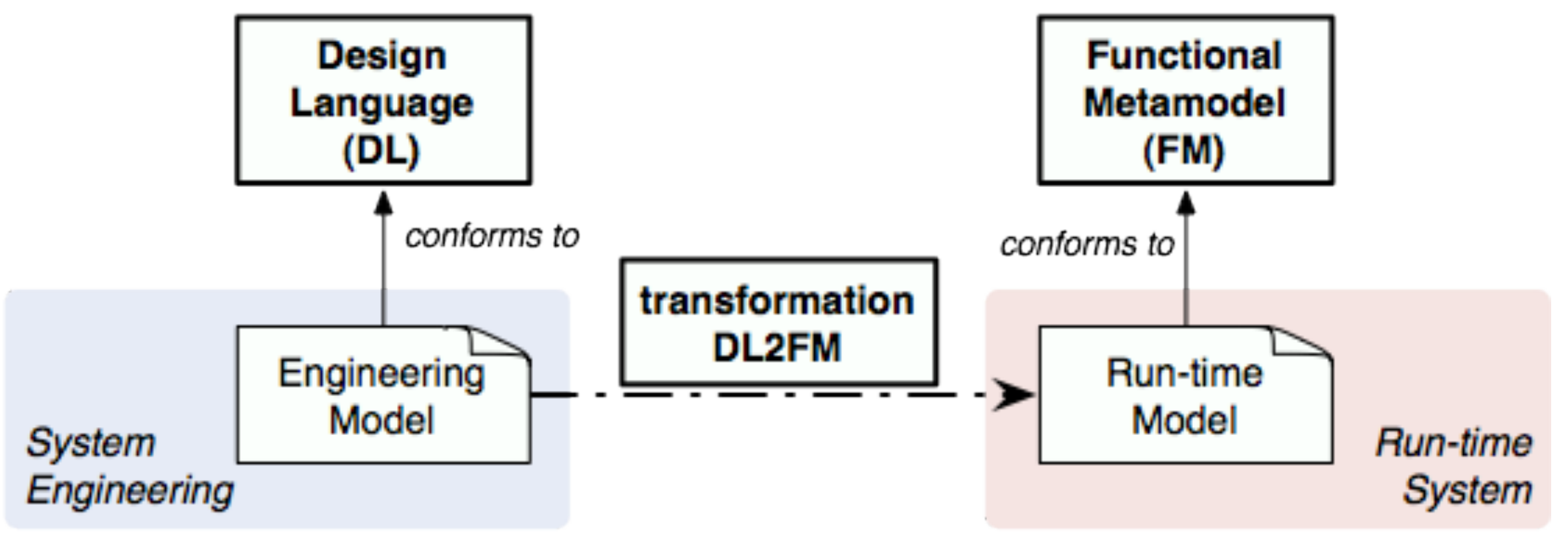




\section{Composing Patterns}

- Patterns can be composed to generate complex architectures

E.g. composing the ECL and the $M C$

Implements the $\mathrm{MC}$ layer as another ECL

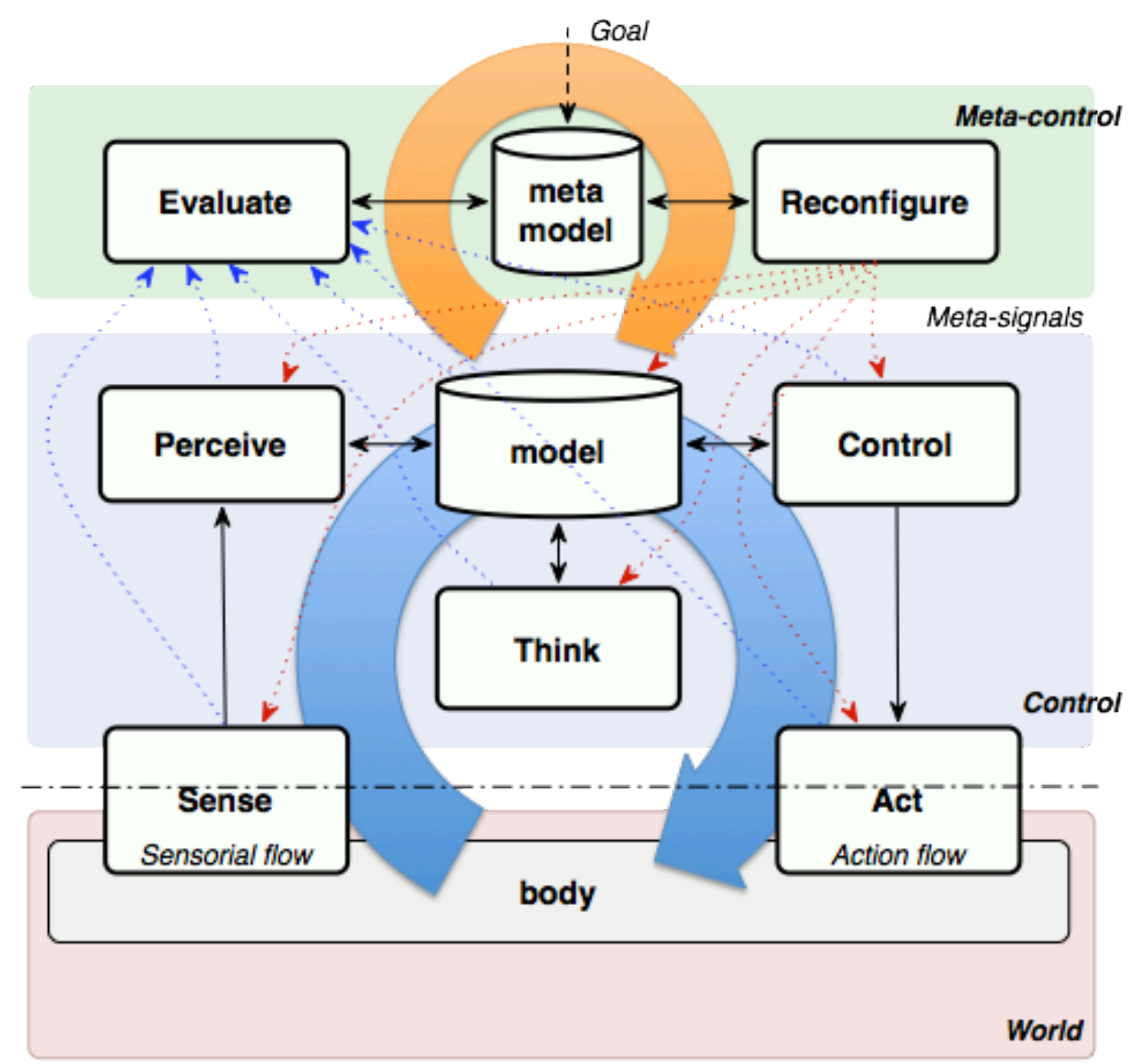




\section{Patterns may be explanatory}

They can be used to capture, describe and analize biological system organization

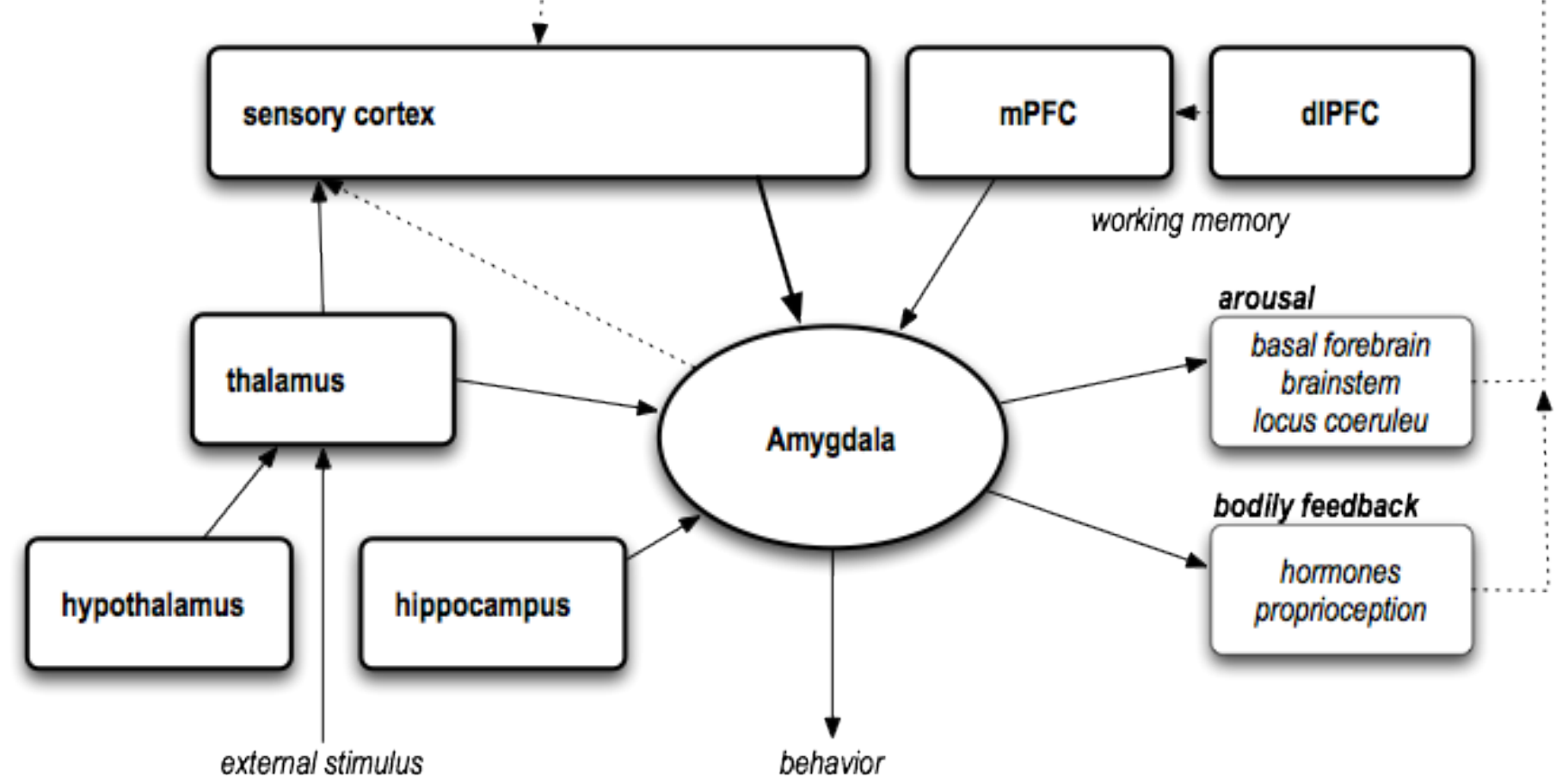

[Arbib-Fellous] 


\section{The Emotion Pattern}

A double ECL

- An architectural model of emotion

Emotion as patterned metacontrol

- Presented at BICA last year

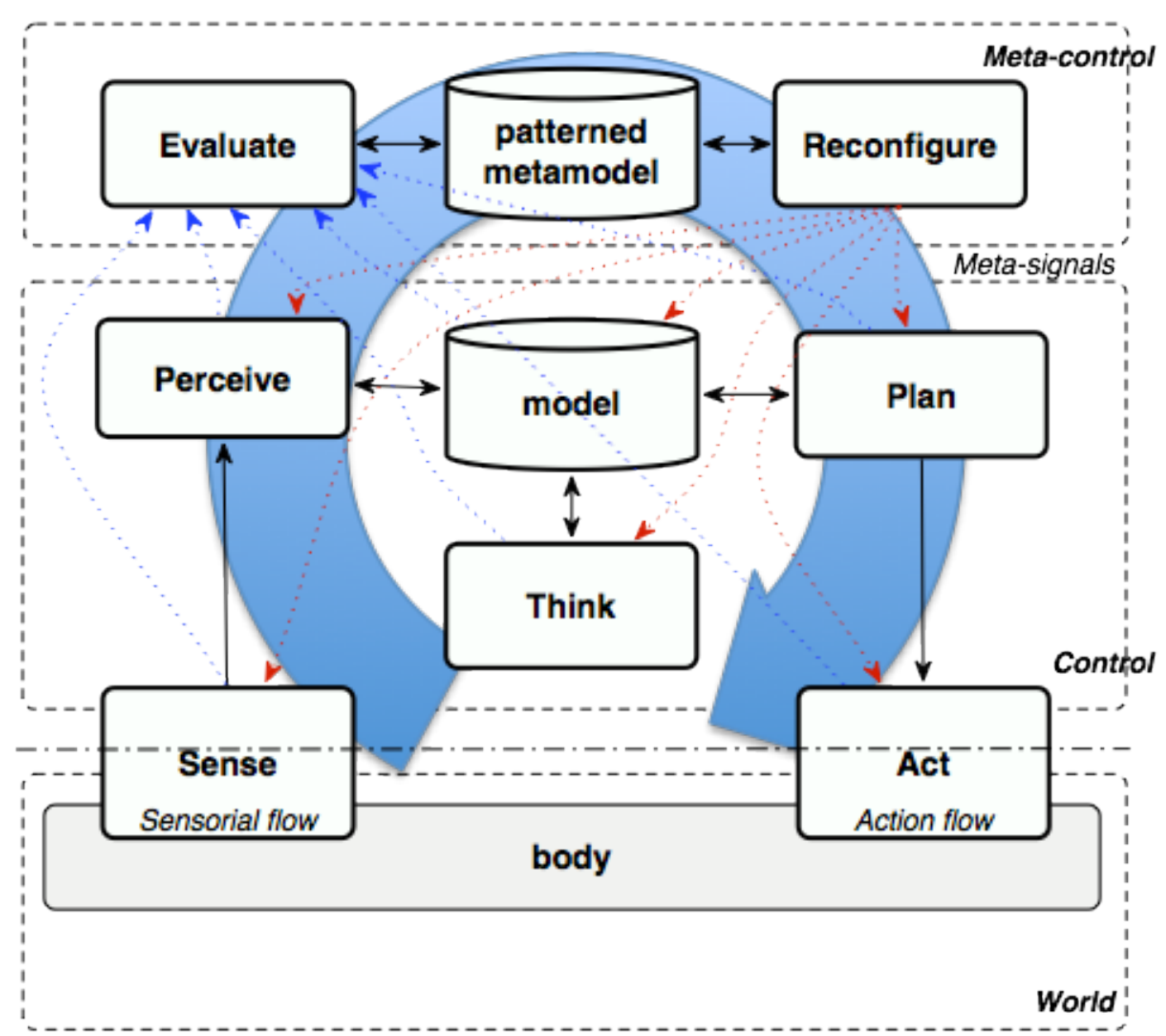


A full agent architecture under development

- Exploits the double ECL emotional pattern, the Layers pattern and multiple Broker patterns

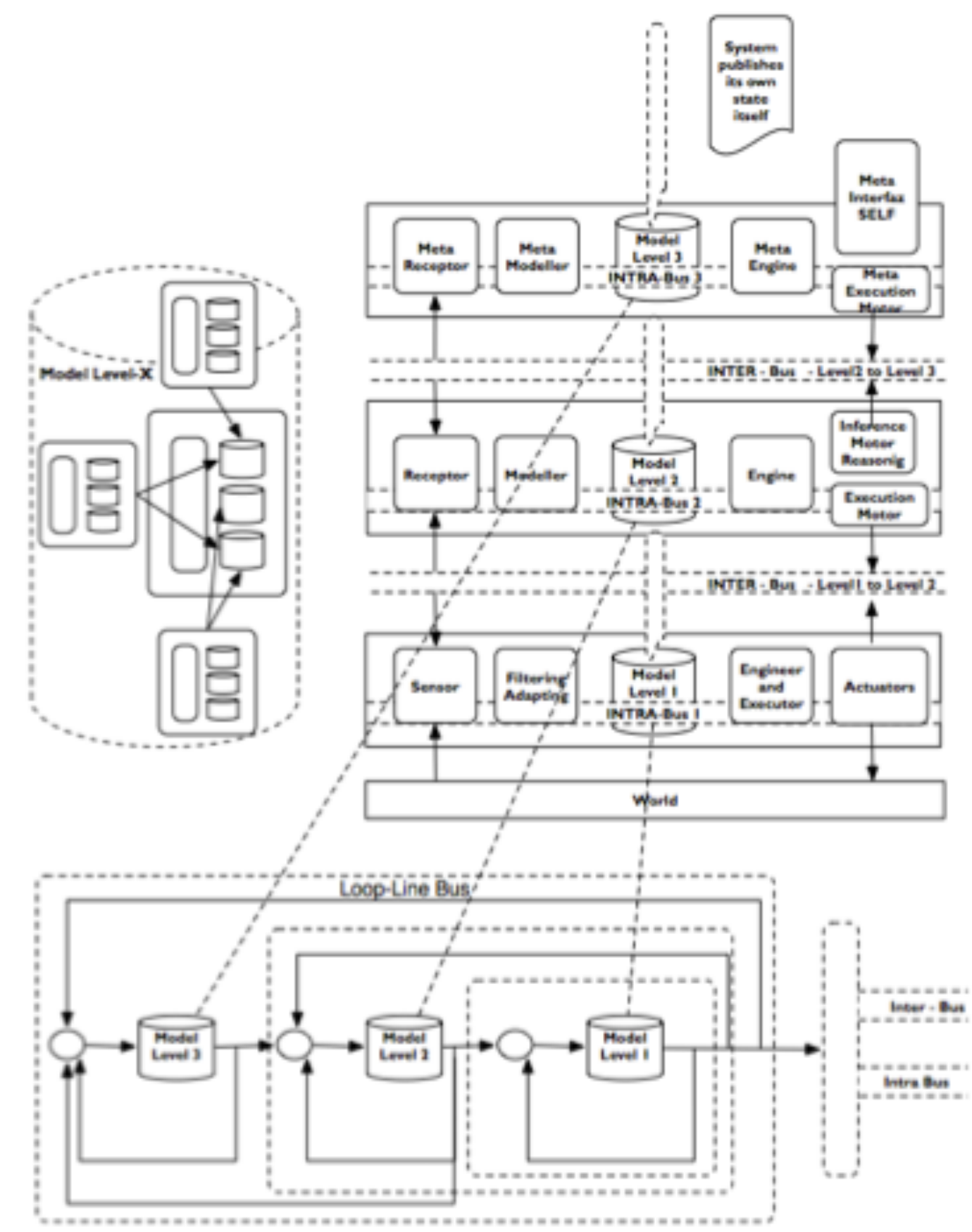




\section{Emotion and Function}

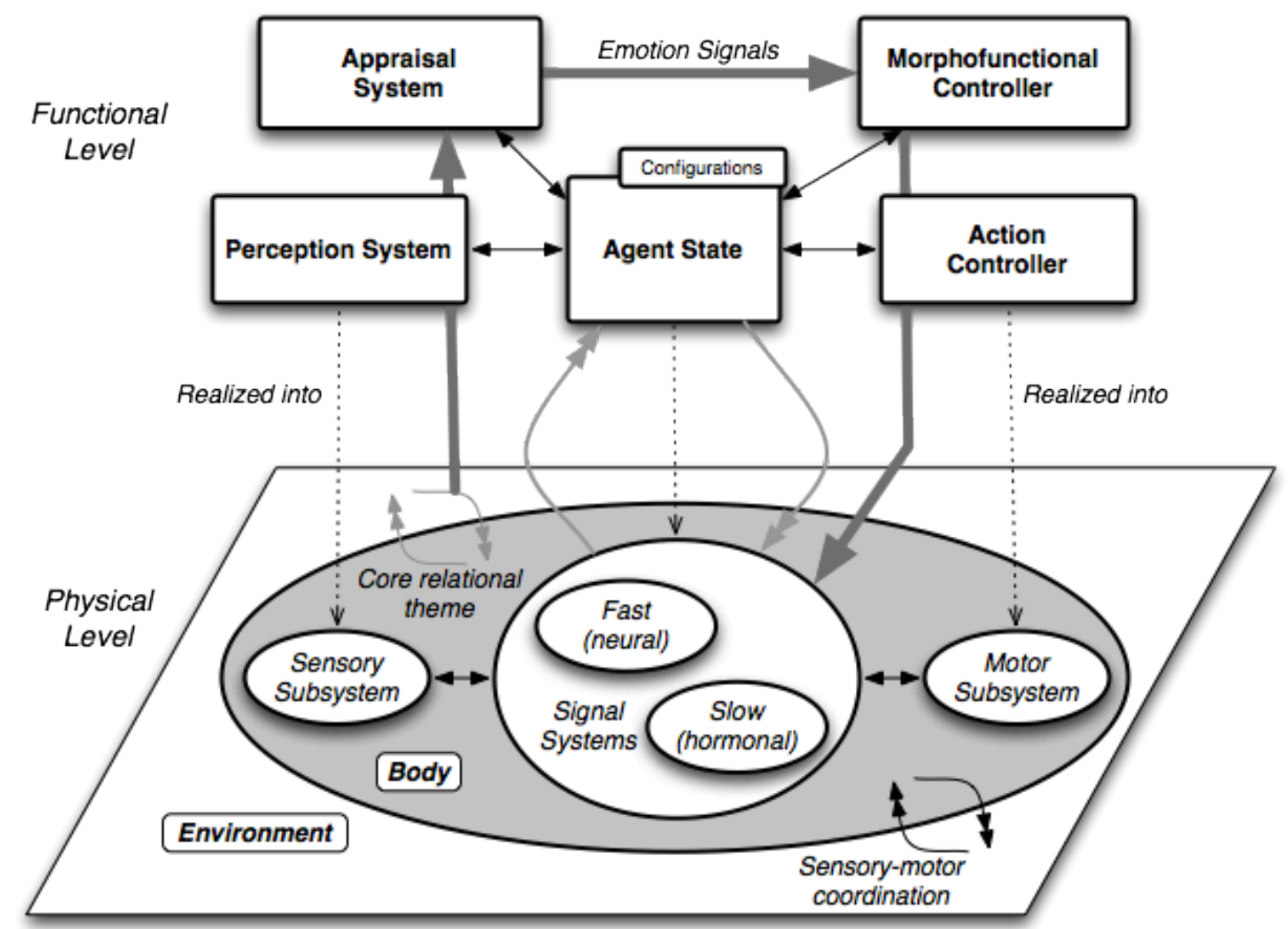

$E C L$ and $M C$ as explanation of Morphofunctionality 


\section{Example Implementation}




\section{The Operative Mind}

An implemented architecture for robots

Follow the Self-Awareness Principle

System Self-awareness: A system is conscious if it is continuously generating meaning from continuously updated self-models.

- Consciousness as a model-based operating system:

- Set of services that manage the structure of the control system to adapt it to the objectives. For that a model of the control architecture is used. 


\section{Exemplary System: mobile robot}

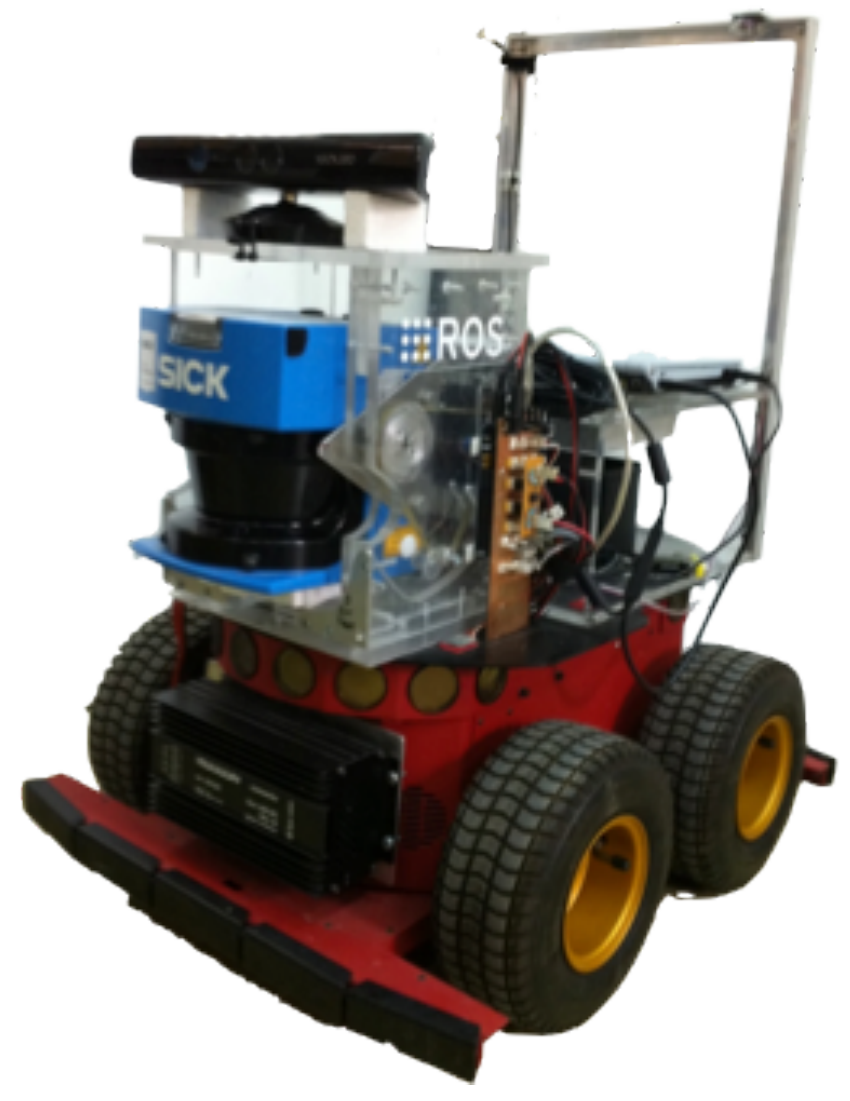

System

variable of interest: position

Mission

objective: navigate to a destination

- Environment

- laboratory: perform SLAM

- Realization

ROS stacks 


\section{Domain Control System}

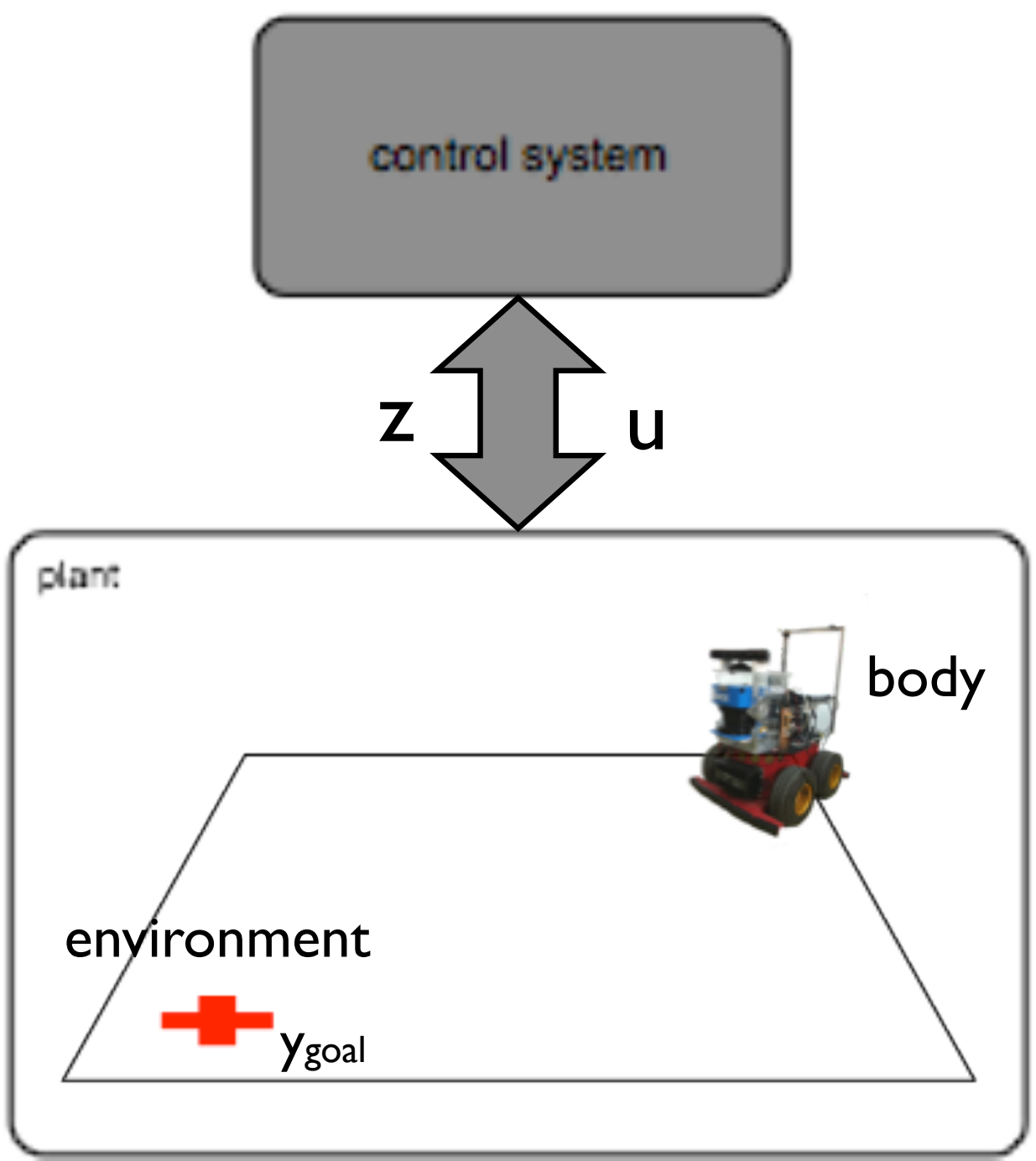

Controlled system (plant)

robot (y)

action / sensing

$\mathrm{u}=(\mathrm{v}, \mathrm{w}) ; \mathrm{z}=$ (odom, laser)

- Obtain a desired behaviour (system's requirements)

$y=y_{\text {goal }}$

constraints on $y(t)$

constraints on $u(t)$ 


\section{A Global vision of OM}

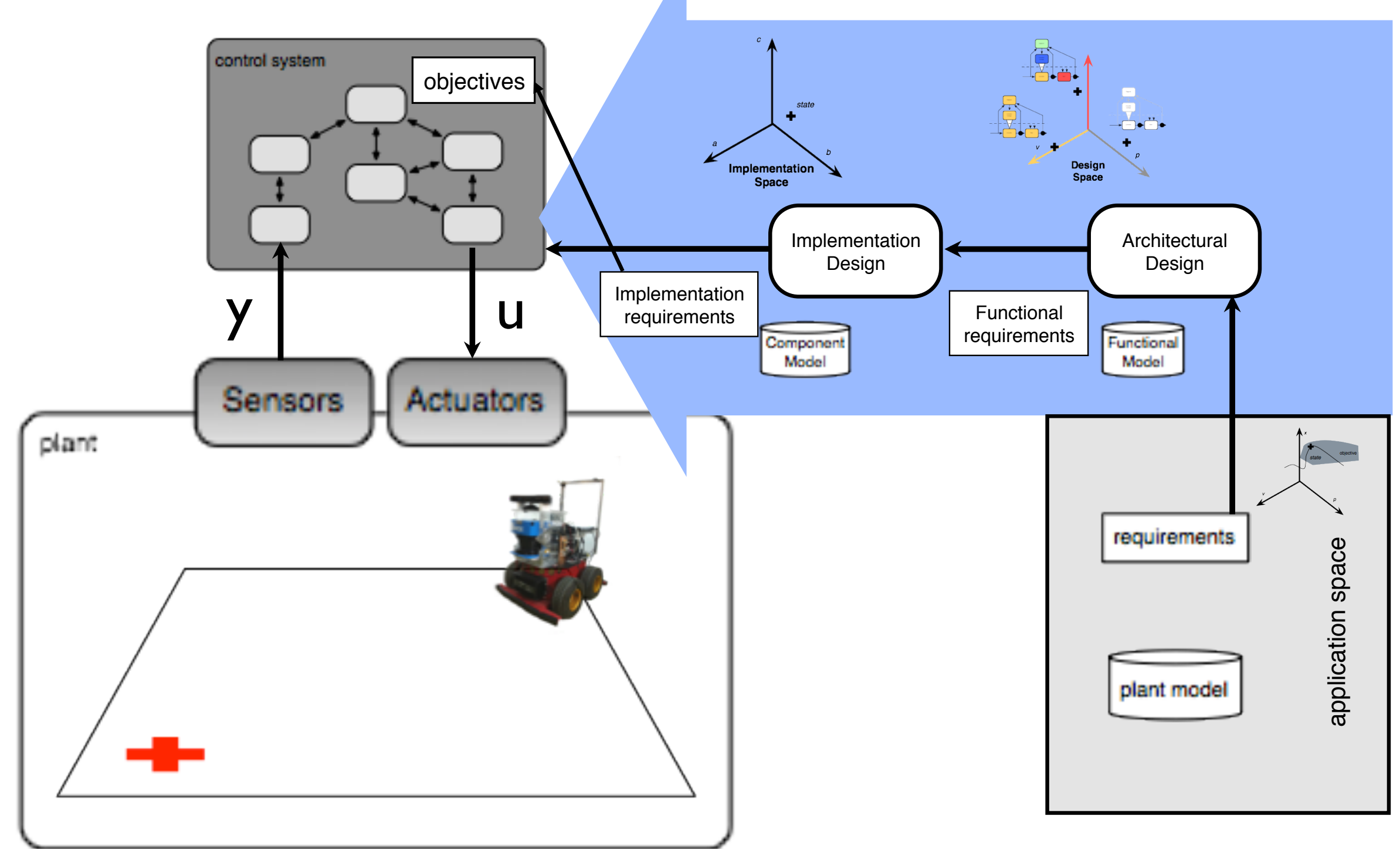




\section{Domains/Spaces involved}

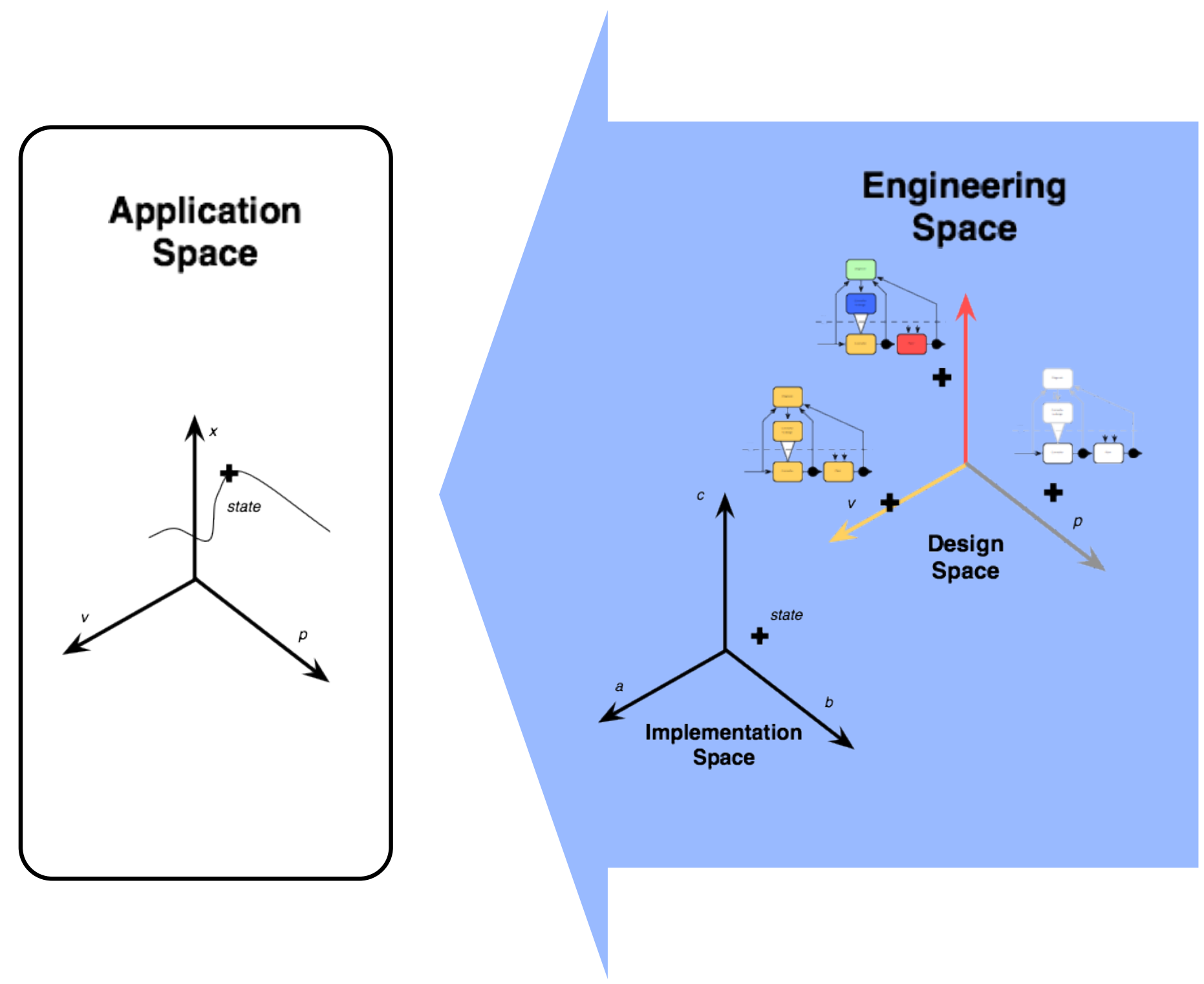




\section{Basic Objective}

Realize a fault-tolerant control

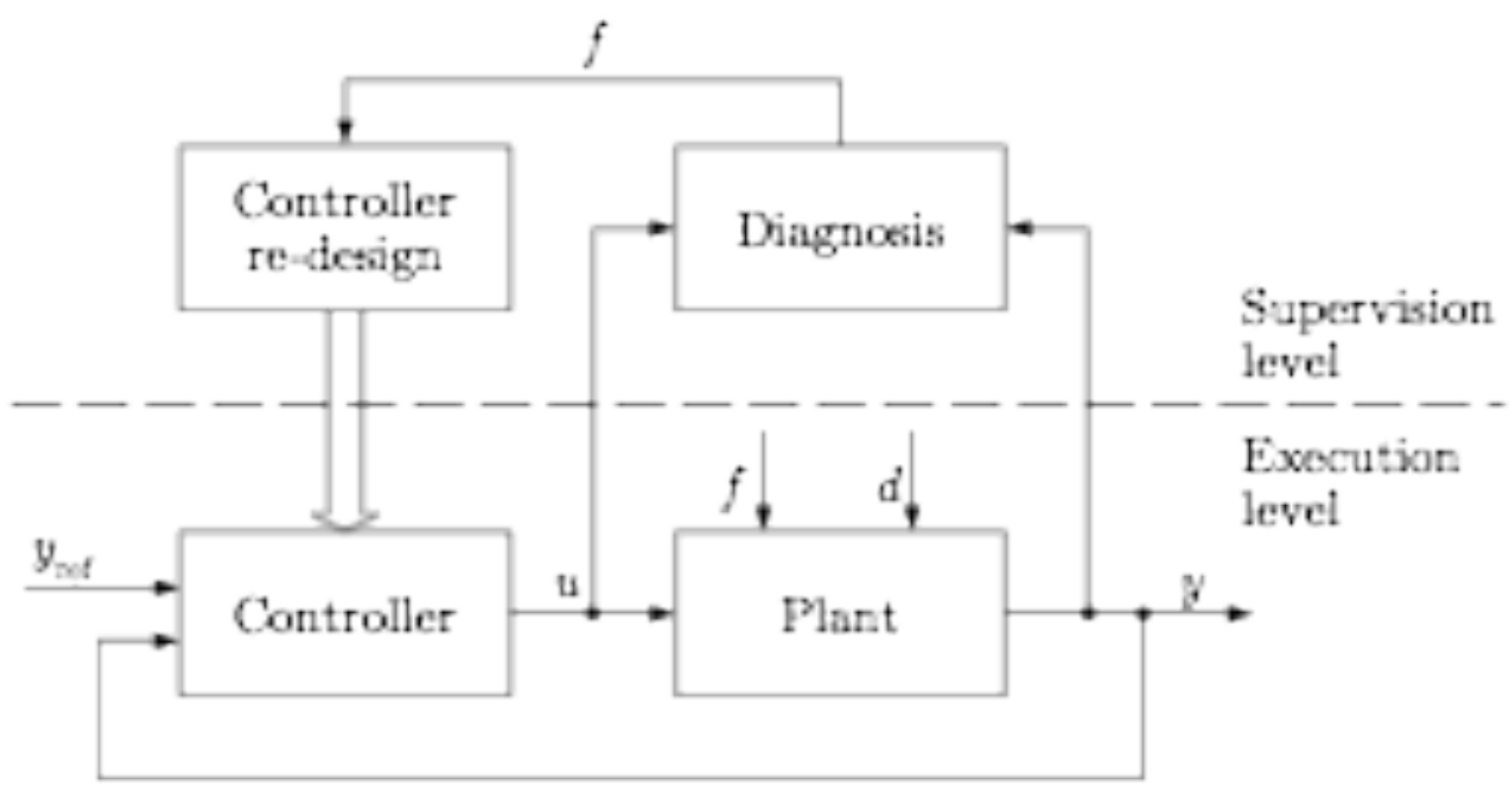




\section{Overall View of OM}

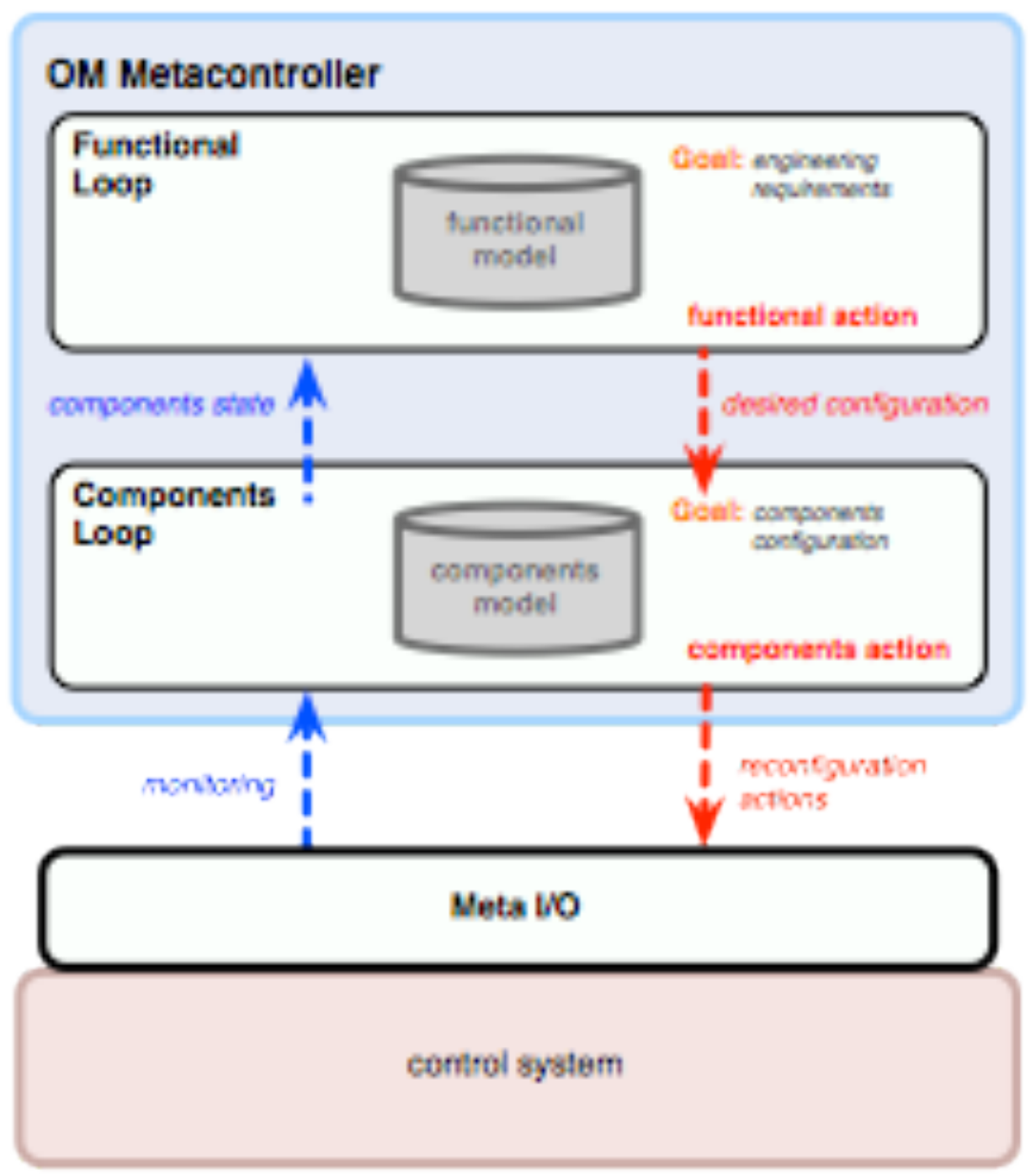




\section{The OM Core Design}

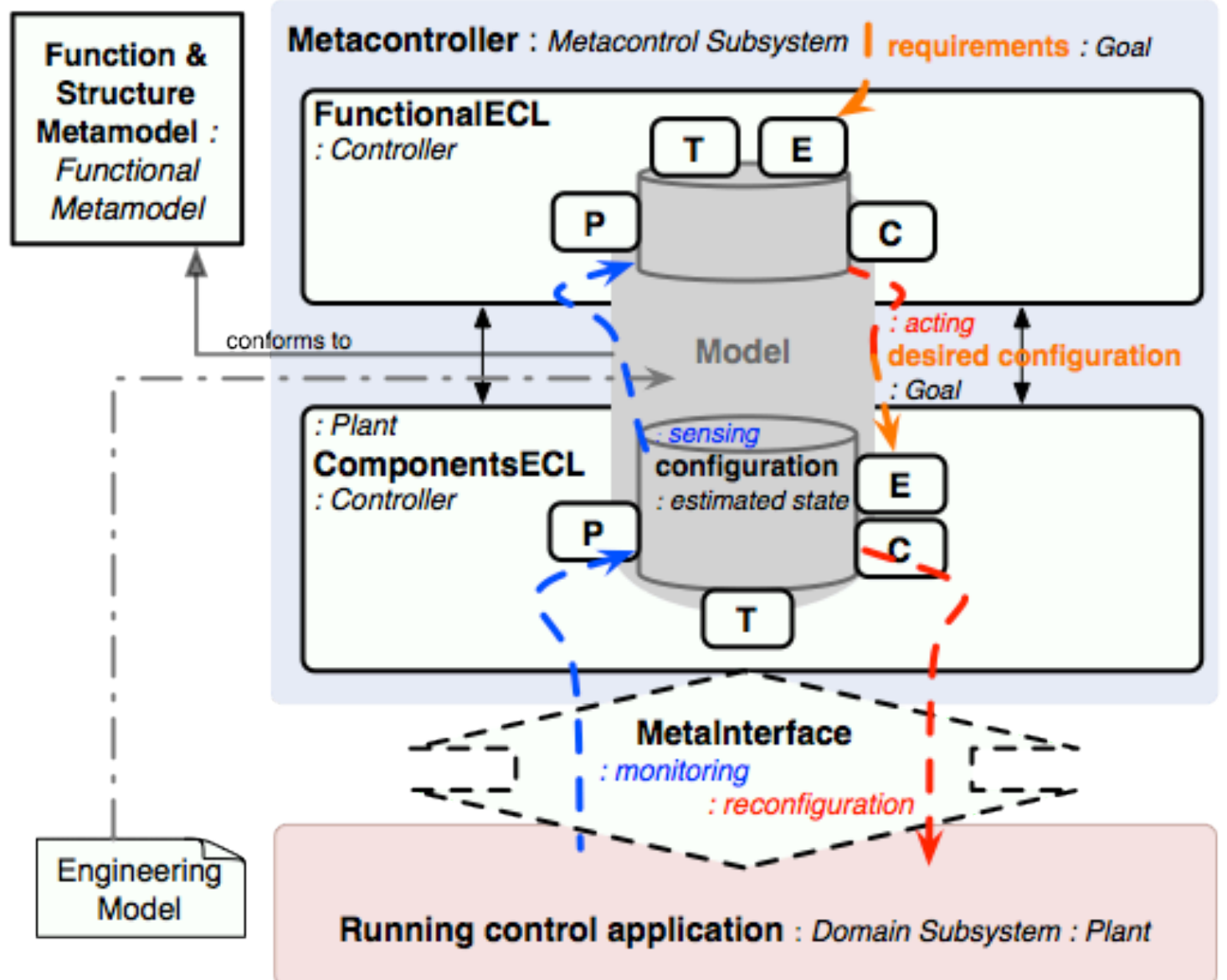

- A double ECL

ECL at the functional level (how functions attain system goals)

ECL at the component level (how component organizations provide functions) 


\section{OM Functional Control}

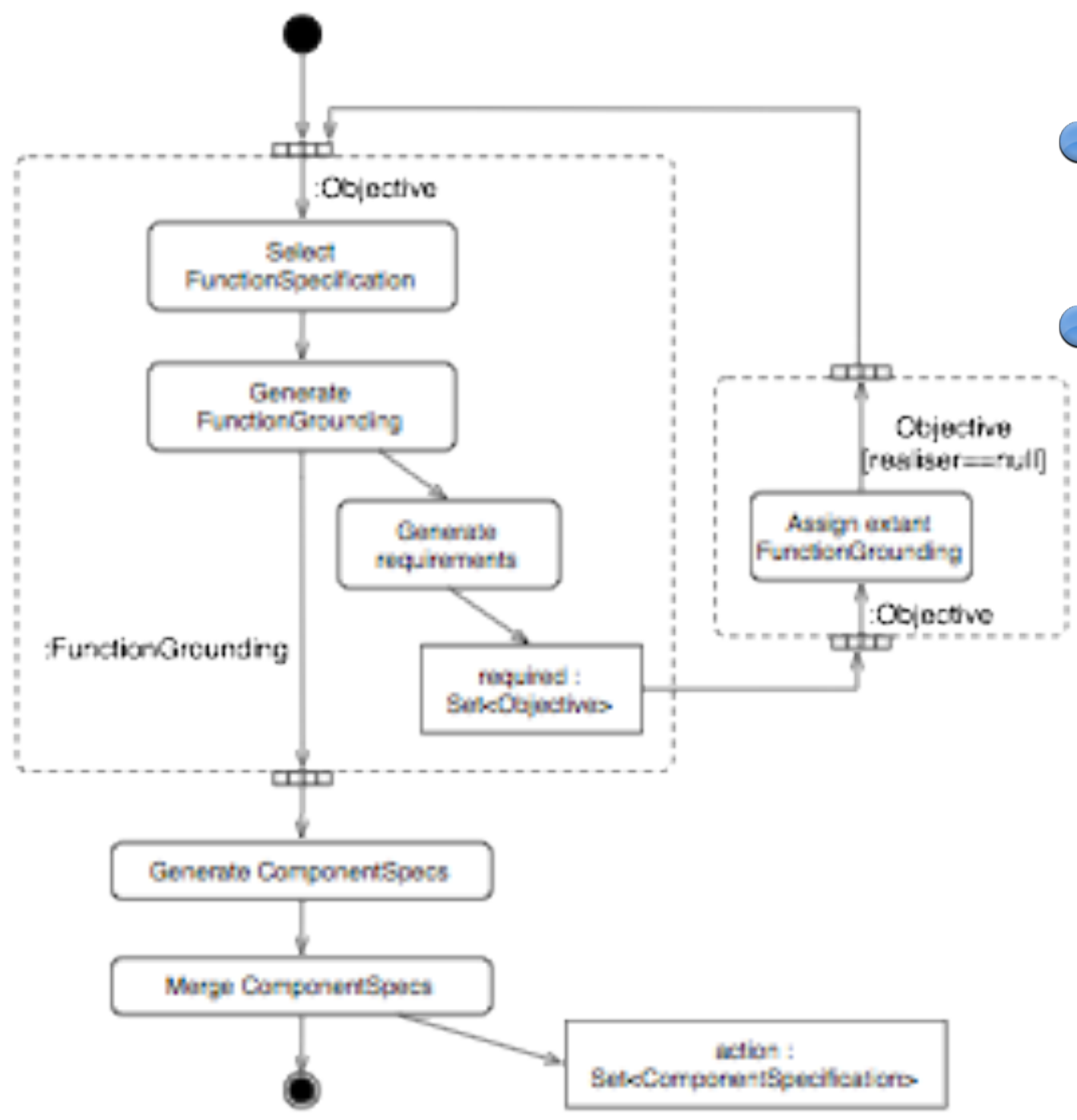

- Core control process at the functional level

- It exploits at run-time the OM FunctionStructure Metamodel to reason about component realizations of functions 


\section{OM Development Process}

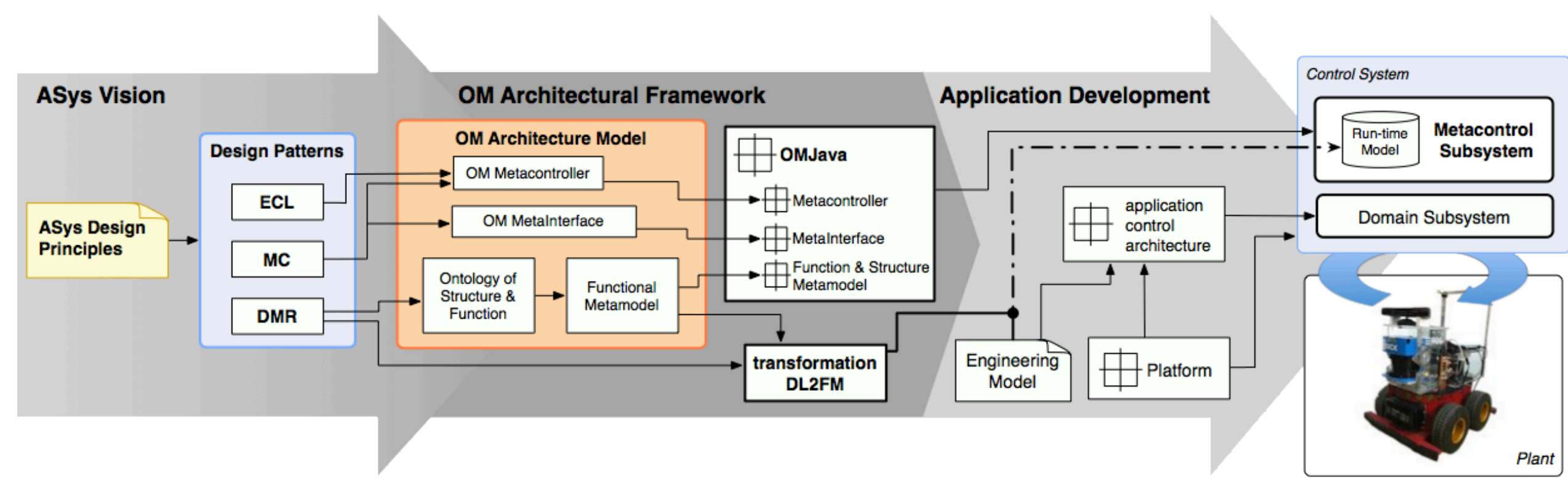




\section{Conclusions}




\section{Conclusions}

Models enable cognition and, in particular, enable selfcognition.

- Patterns enable the capture of architectural aspects of cognitive systems in a domain independent way.

- Pattern composition can be used to generate complex and even complete architectures

We have presented three patterns that capture basic aspects of cognitive self-awareness: ECL, MC, DMR

- They can be used to model biological aspects -e.g. emotion or morphofunctionality- or to build controllers -e.g. OM for mobile robots. 


\section{Thnks}

Students

- Carlos Hernández

- Guadalupe Sánchez

- Carlos Herrera

Funding

European Commission through grants ICEA and HUMANOBS 


\section{References}

- A. Saffiotti, M. Broxvall, M. Gritti, K. LeBlanc, R. Lundh, J. Rashid, B. Seo, and Y. Cho, "The PEIS-ecology project: vision and results," in Proc of the IEEE/RSJ Int Conf on Intelligent Robots and Systems (IROS), Nice, France, 2008, pp. 2329-2335.

- Albus, J. S. (1995). RCS: A Reference Model Architecture for Intelligent Systems. In AAAI 1995 Spring Symposium on Lessons Learned from Implemented Software Architectures for Physical Agents. 


\section{Towards \\ Architectural Foundations for Cognitive Self-aware Systems}

Ricardo Sanz

Universidad Politécnica de Madrid Autonomous Systems Laboratory
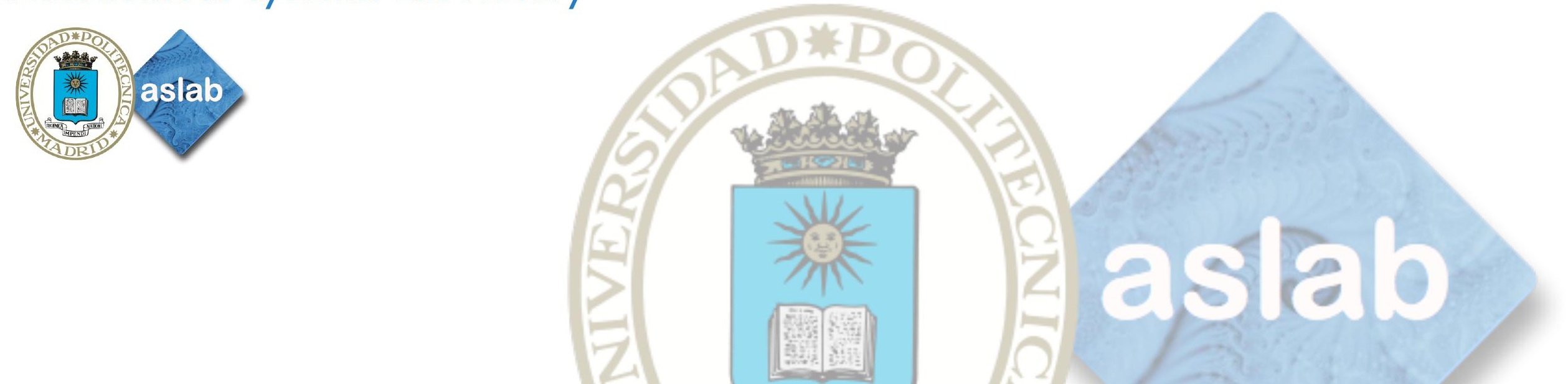